\title{
QUALITY ASSURANCE OF DOSE DISTRIBUTION OF PHOTON BEAM PLANNING BY ANISOTROPIC ANALYTICAL ALGORITHM (AAA)
}

\author{
M Jahangir Alam, Syed Md Akram Hussain ${ }^{1}$, Kamila Afroj ${ }^{2}$ \\ and Shyam Kishore Shrivastava ${ }^{3}$ \\ Department of Biomedical Physics \& Technology, University of Dhaka, Bangladesh \\ email: meos.bd@ @otmail.com
}

\begin{abstract}
A three dimensional treatment planning system has been installed in the Oncology Center, Bangladesh. This system is based on the Anisotropic Analytical Algorithm (AAA). The aim of this study is to verify the validity of photon dose distribution which is calculated by this treatment planning system by comparing it with measured photon beam data in real water phantom. To do this verification, a quality assurance program, consisting of six tests, was performed. In this program, both the calculated output factors and dose at different conditions were compared with the measurement. As a result of that comparison, we found that the calculated output factor was in excellent agreement with the measured factors. Doses at depths beyond the depth of maximum dose calculated on-axis or off-axis in both the fields or penumbra region were found in good agreement with the measured dose under all conditions of energy, SSD and field size, for open and wedged fields. In the build up region, calculated and measured doses only agree (with a difference $2.0 \%$ ) for field sizes $>5 \times 5 \mathrm{~cm}^{2}$ up to $25 \times 25 \mathrm{~cm}^{2}$. For smaller fields, the difference was higher than $2.0 \%$ because of the difficulty in dosimetry in that region. Dose calculation using treatment planning system based on the Anisotropic Analytical Algorithm (AAA) is accurate enough for clinical use except when calculating dose at depths above maximum dose for small field size.
\end{abstract}

Keywords: Treatment Planning, Three dimensional treatment plan, Radiotherapy, Anisotropic Analytical Algorithm, Photon dose distribution

\section{INTRODUCTION}

The radiation treatment planning process is complex and involves multiple steps and a number of technologies. The first step in the process includes the derivation of patient anatomical information. This information is then used to determine the location of the tumor and important normal tissues that could be affected by the radiation treatment. The TPS is used to determine the dose distribution that will result in the body from selected incident beams [1]. The optimum beam arrangement that will provide adequate coverage of the malignant tissues while minimizing the dose to critical normal tissues will be selected. Advances in computer technology have led to the availability of sophisticated three dimensional (3D) Treatment Planning Systems (TPS) for use in many radiotherapy centers. One aim of introducing such sophisticated 3D TPS is to improve the accuracy of dose calculations in radiotherapy planning. The accuracy in radiation therapy has been discussed in several reports [2], which showed that errors in dose delivery should not exceed $2.0 \%$. Values as low as $0.3 \%$ have, however, been also mentioned. To investigate the accuracy of the TPS, several quality assurance (QA) programs have been introduced and discussed in the literature. These QA programs mainly check the agreement of the output data from the system with the measured data. A new 3D-TPS (Treatment Management System-TMS version 8.6, VMS, Varian Medical System USA) was installed in the Oncology Center, Dhaka. This TPS is based on the Anisotropic Analytical Algorithm (AAA), where physical quantities, estimated using conventional measured quantities, are used. The photon dose calculation model in this treatment planning system is based on a convolution algorithm. Briefly, the model is used to compute weight for depth dose curves

\footnotetext{
${ }^{1}$ Department of Radiation Oncology, BSMMU, Shahbag Dhaka, Bangladesh

${ }^{2}$ Institute of Nuclear Medicine \& Ultrasound, Bangladesh Atomic Energy Commission, Dhaka.

${ }^{3}$ Department of Radiation Oncology Tata Memorial Hospital, Parel, Mumbai 400012, India
} 
from monoenergetic photons [3]. These monoenergetic depth doses, calculated with the convolution method from Mont Carlo generated point spread functions (PSF), are added to yield the pure photon depth dose distribution. Poly-energetic pencil-beam is then used to calculate the dose distribution for a given case by convolution with the machine specific energy-fluence matrix modulated by the actual field shape. Inversely, the energy fluence matrix is obtained as a convolution of the pencil-beam dose distribution in a reference situation. One of the features of the system is that it calculates the monitor settings for the planned fields. The aim of this study is to verify the validity of the calculated photon beam data in comparison with the measured photon data at different treatment situations.

\section{METHODS AND MATERIALS}

To characterize a particular 6MV Photon beam in TMS, the measurements were performed the centralaxis depth doses at field sizes $5 \times 5,10 \times 10,15 \times 15$ and $20 \times 20$, all in units of $\mathrm{cm}^{2}$, at $90 \mathrm{~cm}$ source surface distance ( SSD). These depth dose data are used in determination of energy spectrum and modeling of contaminating electrons. In-plane and cross-plane the dose profiles was measured at depths of 1.5, 5, 10, 15 and $20 \mathrm{~cm}$ at $90 \mathrm{~cm} \mathrm{SSD}$ in water for the field sizes of $5 \times 5 \mathrm{~cm}^{2}, 10 \times 10 \mathrm{~cm}^{2}, 15 \times 15 \mathrm{~cm}^{2}$ and $20 \times 20$ $\mathrm{cm}^{2}$. These beam profiles data are used for penumbra modeling (i.e. source size and distribution modeling) and modeling of contaminating photons outside the geometric field. The star shaped dose profiles at the reference $90 \mathrm{~cm} \mathrm{SSD}$ and $10 \mathrm{~cm}$ depths were using a maximum collimator setting. These star profiles are used to calculate energy fluence distribution and for fluence modulation vector for wedges. Collimator head scattering factors and output factors were measured in water for open and wedged fields at the reference SSD and depth using different field sizes. These factors are used to monitor unit calculations and output factor (absolute dose) in air at calibration geometry for different field sizes. These data were also used to monitor unit calculation. All of these measurements were performed for both open and wedge fields by using the following equation. The absorbed dose to water at the reference depth $\mathrm{Z}_{\mathrm{ref}}$ in water, in a photon of beam quality $\mathrm{Q}$ and in the absence of the chamber, is given by

$$
D_{W, Q}=M_{Q} \times N_{D, W} \times Q_{0} \times k_{Q, Q_{0}}
$$

where $\mathrm{M}_{\mathrm{Q}}$ is the reading of the dosimeter with the reference point of the chamber positioned at $\mathrm{z}_{\mathrm{ref}}$ in accordance with the reference conditions and corrected for the influence quantities temperature and pressure, electrometer calibration, polarity effect and ion recombination. $\mathrm{N}_{\mathrm{D}, \mathrm{w}}, \mathrm{Q}_{0}$ is the calibration factor in terms of absorbed dose to water for the dosimeter at the reference quality $\mathrm{Q}_{0}$, and $\mathrm{K}_{\mathrm{Q}, \mathrm{Q} 0}$ is a chamber specific factor which corrects for difference between the reference beam quality $\mathrm{Q}_{0}$ and the actual quality being used, Q. All measured depth dose curves and beam profiles (including star profiles) were scanned using Scanditonix and IBA RFA 300 radiation field analyzer using $0.1 \mathrm{cc}$, IC10 Welhofer ionization chamber and CC 113,114 \& FC 65. Absolute and relative output factors were measured using Farmer dosimeter with $0.6 \mathrm{cc}$ graphite guarded stem ionization chamber from IBA. For measurements in air, the ion chamber was covered by a brass cap to reduce electron contamination and to achieve a proper build up of secondary particles. Two linear accelerators were used in this study. One accelerator is Siemens Mevatron 7445 that has only $6 \mathrm{MV}$ photon beam and the other accelerator is Siemens Primus II for 6MV and $10 \mathrm{MV}$ photon beams respectively. These two accelerators are provided with fixed wedges of $15^{\circ}$, $30^{\circ}, 45^{\circ}$ and $60^{\circ}$. Information regarding the treatment machines is supplied to the planning system. The information is treatment head geometry; the distances from the target to each of the flattening filter, monitor chamber, upper and lower collimators, wedge, block tray and isocenter. It includes also the thickness of both lower and upper collimators [3]. These five sets of data are stored in data files. To verify the photon beam data calculated by TMS, a QA program consisting of the following tests was performed.

Investigating the data files of the two linear accelerators which include the data mentioned in the previous paragraph to check for possible bugs in the implementation. This was done by performing some demo 
treatment plans using different techniques. In these techniques all data files have been used. Then the values in the data files were compared with the output in a calculation performed by the TPS and evaluating the performance of the system in calculating the absolute dose. The absolute doses at $10 \times 10$

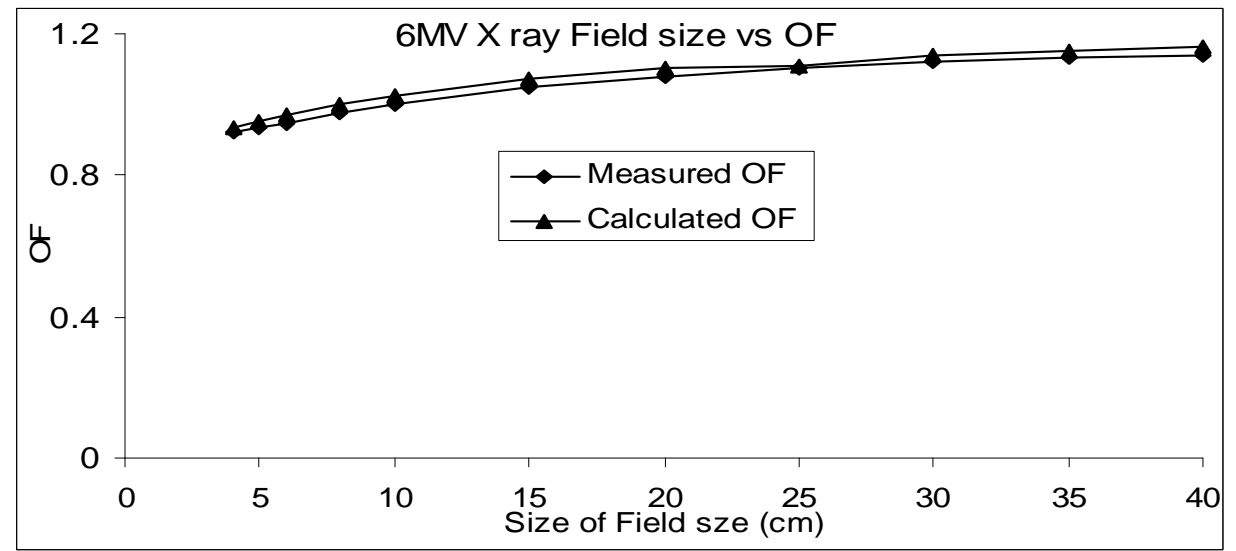

Fig. 1: Normalized output factor (output factor divided by output factor of $10 \times 10 \mathrm{~cm}^{2}$ field) for open fields of $6 \mathrm{MeV}$ photon generated by Mevatron 7445 plotted against field size. The line represents the measured output factors and point represents the calculated factors.

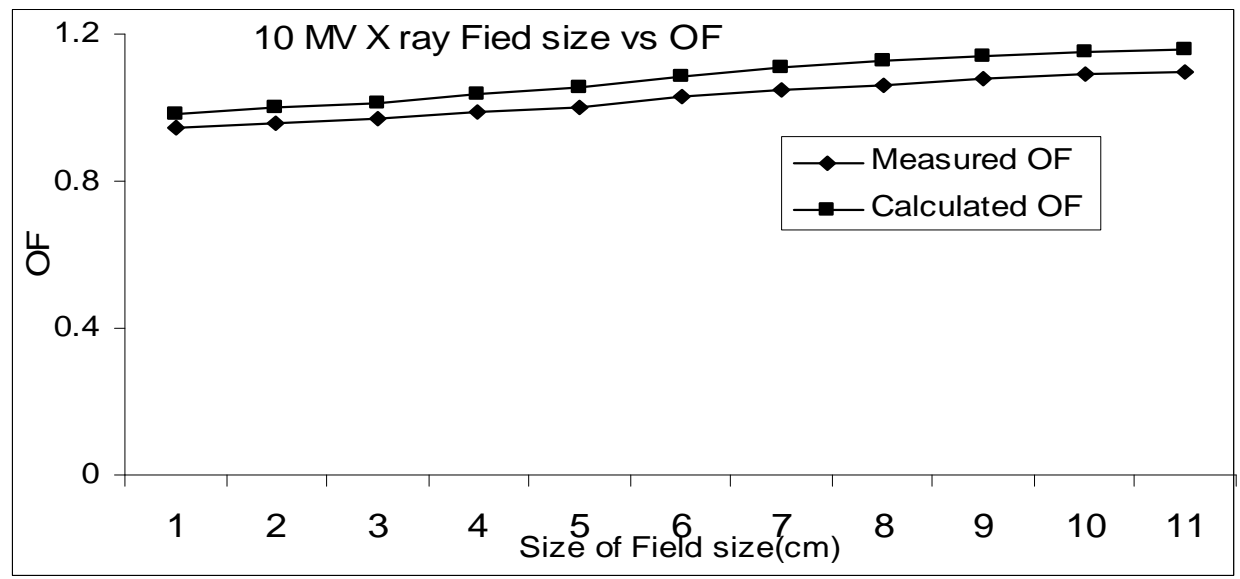

Fig. 2: Normalized output factor (output factor divided by output factor of $10 \times 10 \mathrm{~cm}^{2}$ field) for $45^{\circ}$ wedged fields of $10 \mathrm{MeV}$ photon generated by Mevatron 7445 plotted against filed size. The line represents the measured output factors and point represents the calculated factors.

$\mathrm{cm}^{2}$ for both open and wedged fields at the reference positions, as described above, were measured. These measured doses were compared to the output calculated by TMS for the corresponding situations. In this test the output factors at reference SSD and depth were calculated for different field sizes from $5 \times 5 \mathrm{~cm}^{2}$ up to the maximum field size $\left(40 \times 40 \mathrm{~cm}^{2}\right)$. The output factor for a certain field size was considered as the dose per monitor unit at the reference SSD and depth on the central axis divided by the corresponding value for the $10 \times 10 \mathrm{~cm}^{2}$ field. This test investigates the ability of the system to reproduce the input data used during implementation of the machines. In this test the depth doses and doe profiles mentioned above were calculated and compared with measured data[4]. To check the energy fluence modulation matrix, the star profiles measured at reference SSD and depth for maximum field sizes in both open and wedged fields were compared with the corresponding calculated star profiles. In the last test depth dose 
lines calculated at different SSDs from 80 to $100 \mathrm{~cm}$ using $10 \times 10 \mathrm{~cm}^{2}$ field size for both open and wedged fields were compared with the corresponding measured depth dose lines.

\section{RESULTS AND DISCUSSION}

The information in the data files of the two accelerators were found to agree in all aspects with the output data obtained from the TPS. The absolute doses calculated at the reference point for the three photon beams were in agreement with measurements for both open and wedged fields. The errors were within the limit of truncation errors. The calculated output factors are showing good agreement with the measured values.

For open fields using both energies from the two linear accelerators the error in calculated normalized out put factor did not exceed $\pm 0.0021( \pm 0.21 \%)$ compared to measured values. Fig. (1) shows an example of this result for open fields of $6 \mathrm{Mev}$ photon from Mevatron 7445. For wedged fields, the error was slightly

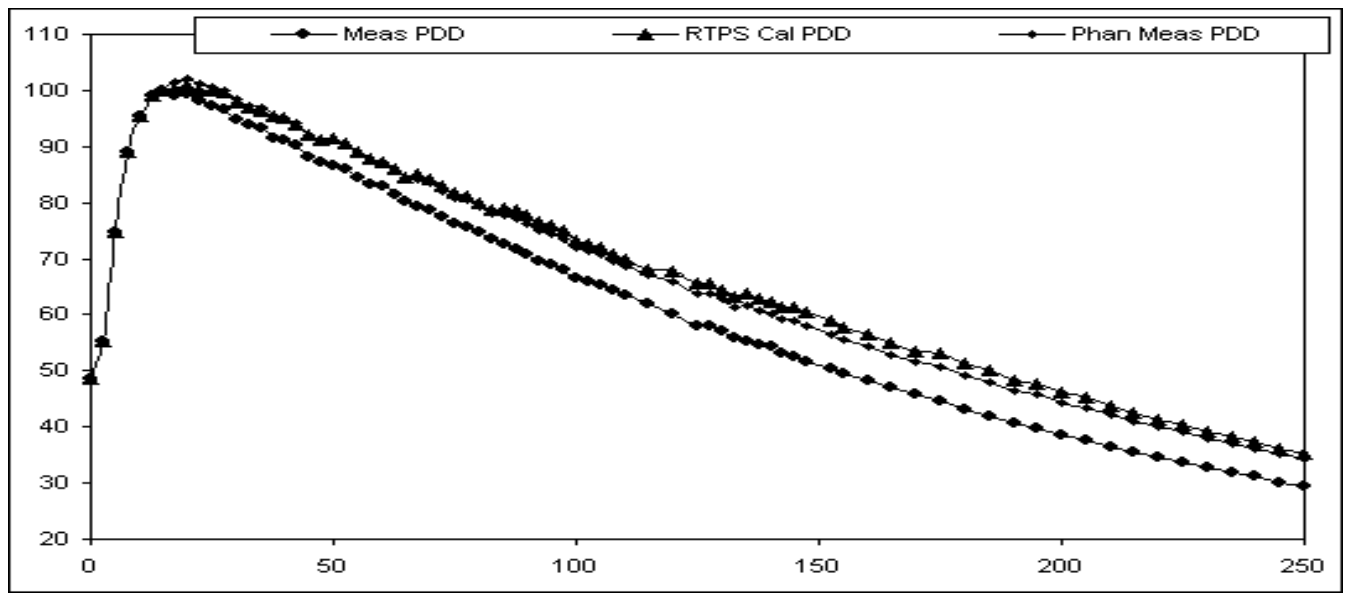

Fig. 3: An example of comparison between measured and calculated depth dose data of $6 \mathrm{MV}$ photons generated by Mevatron 7445 linear accelerator at $90 \mathrm{~cm}$ SSD with 4 different field sizes $(5 \times 5,10 \times 0,15 \times 15$ and $20 \times 20 \mathrm{~cm}^{2}$ ). The depth doses divided by monitor unit is normalized at $10 \times 10 \mathrm{~cm}^{2}$ field. The dotted lines represent calculated data and solid lines represent measured data

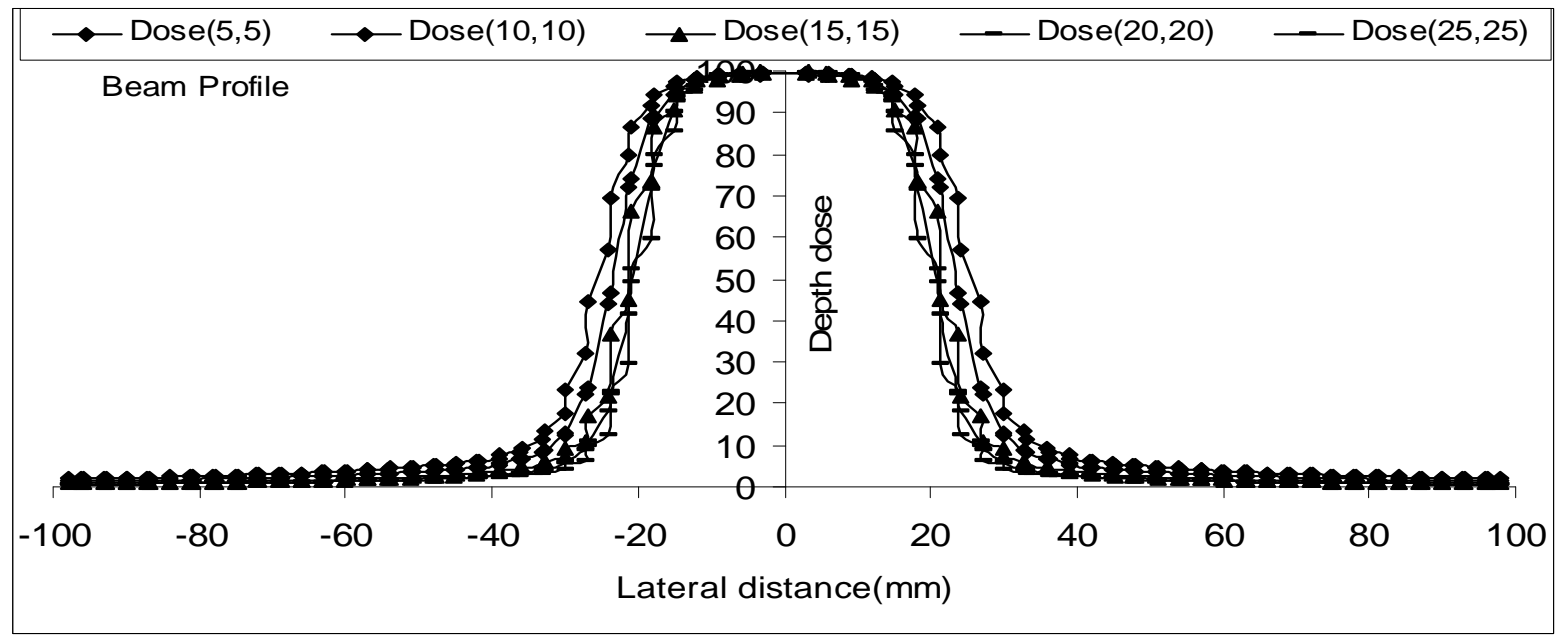

Fig. 4: An example of comparison between measured and calculated beam profiles of 10MV photons generated by Primus II linear accelerator at $90 \mathrm{~cm}$ SSD with 4 different field sizes $\left(5 \times 5,10 \times 10,15 \times 15\right.$ and $\left.20 \times 20 \mathrm{~cm}^{2}\right)$. The doses divided by monitor unit are normalized at $10 \times 10 \mathrm{~cm}^{2}$ field. The dotted lines represent calculated data and solid lines represent measured data. 
higher than that of open fields. The maximum difference between measured and calculated normalized output factor in case of wedged field was about $0.001(1 \%)$. This maximum error occur in the maximum field size of $10 \mathrm{MeV}$ photon from Mevatron 7445 for $60^{\circ}$ wedge. Fig. (2) shows an example of the comparison between calculated and measured normalized output factors for wedged $10 \mathrm{MV}$ photon fields.

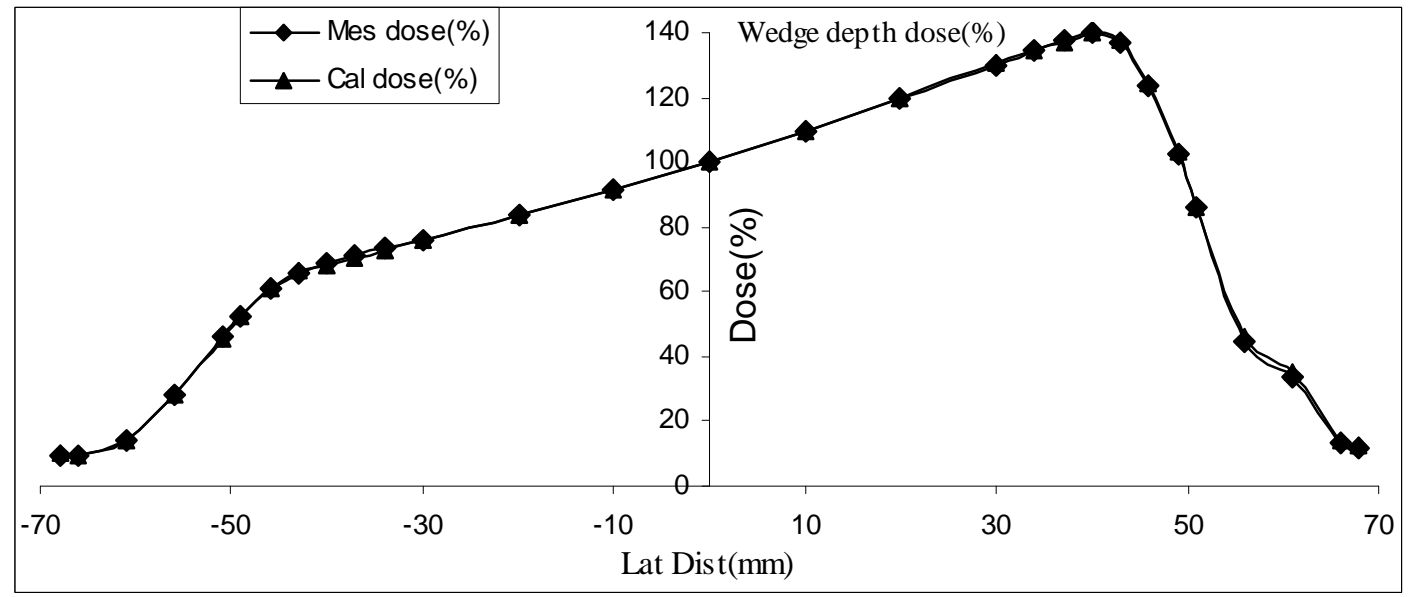

Fig. 5: An example of comparison between measured and calculated beam star profiles of $6 \mathrm{MV}$ photons generated by Primus II linear accelerator at $90 \mathrm{~cm} \mathrm{SSD} \mathrm{with} \mathrm{field} \mathrm{size} 20 \times 20 \mathrm{~cm}^{2}$ using $60^{\circ}$ wedge. These lines represent $45^{\circ}$ star angle. The doses divided by monitor unit are normalized central axis dose of the same field. The dotted line represents calculated data and solid line represents measured data.

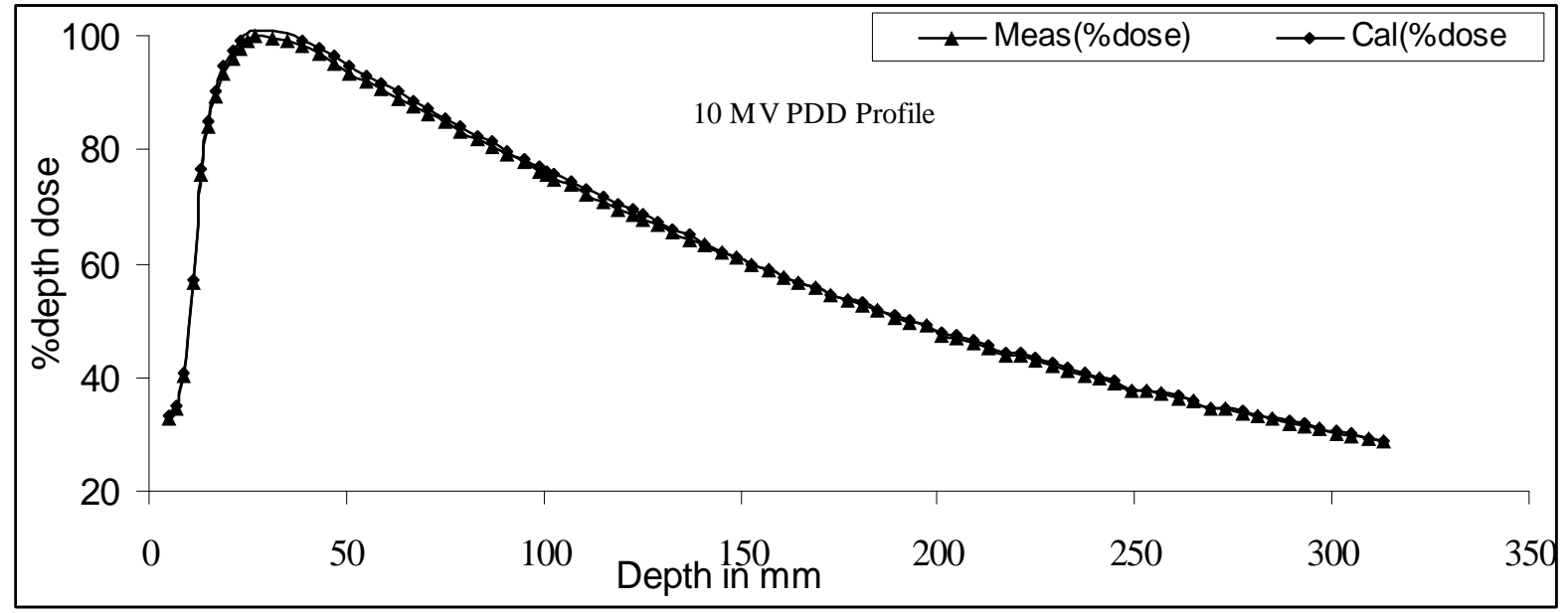

Fig. 6: An example of comparison between measured and calculated depth dose data of $10 \mathrm{MU}$ photons generated by Primus II linear accelerator at $100 \mathrm{~cm}$ SSD with $10 \times 10 \mathrm{~cm}^{2}$. The depth doses divided by monitor unit are normalized at $10 \mathrm{~cm}$ depth. The dotted line represents calculated data and solid line represents measured data.

The comparison between calculated and measured depth doses and dose profiles for both open and wedged fields showed a negligible difference at depths greater than the depth of maximum dose for all energies. This difference was always less than $1 \%$ in both directions. In the build-up region this error was higher than that and depends on the field size. For field sizes $10 \times 10 \mathrm{~cm}^{2}$, the difference ranged between 2-3\%; the larger the size the smaller the error and the error also decreased with increasing the field size and ranged between 3-9\%. Examples of the comparison between the calculated and measured depth dose and beam profiles were shown in Figs. (3\&4) respectively. The comparison at depths larger than the depth of the maximum dose showed also an excellent agreement between calculated and measured beam 
profiles for both open and wedged fields with different field sizes in both regions of useful beam and penumbra.

Comparison of calculated star profiles with measured data for wedged fields showed excellent agreement for all energies. In both the useful beam and penumbra regions, the difference was $<1 \%$ in both directions. This agreement means that, for wedged fields, the input data for the energy fluence modulation matrix is accurately reproducible for the two linear accelerators and for all energies[6]. Fig. (5) Shows an example of calculated star profiles when compared to measured star profiles for wedged field. This test compared calculated depth dose lines measured depth dose lines at different SSDs for $10 \times 10 \mathrm{~cm}^{2}$ field sizes. It showed a general agreement between them for different SSDs at depths larger than depth of the maximum dose with difference less than $1 \%$. As found for SSD $90 \mathrm{~cm}$, the difference in the build-up region varies between 2-3\%. Fig. (6) is an example of that comparison.

Tab1e 1: Depth dose variation for 6 and $10 \mathrm{MV}$ photon beams at buildup region and $10 \mathrm{~cm}$ depth in water

\begin{tabular}{|c|c|c|c|c|c|c|c|}
\hline \multicolumn{4}{|c|}{ 6MV } & \multicolumn{5}{|c|}{$10 \mathrm{MV}$} \\
\hline $\begin{array}{c}\text { Depth } \\
(\mathrm{cm})\end{array}$ & $\begin{array}{c}\text { Measured } \\
\text { dose } \\
(\mathrm{cGy} / 100 \mathrm{MU})\end{array}$ & $\begin{array}{c}\text { Calculated } \\
\text { dose } \\
(\mathrm{cGy} / 100 \mathrm{MU})\end{array}$ & $\begin{array}{c}\text { Deviation } \\
(\%)\end{array}$ & $\begin{array}{c}\text { Depth } \\
(\mathrm{cm})\end{array}$ & $\begin{array}{c}\text { Measured } \\
\text { dose } \\
(\mathrm{cGy} / 100 \mathrm{MU}\end{array}$ & $\begin{array}{c}\text { Calculated } \\
\text { dose } \\
(\mathrm{cGy} / 100 \mathrm{MU})\end{array}$ & $\begin{array}{c}\text { Deviatio } \\
\mathrm{n}(\%)\end{array}$ \\
\hline 1.604 & 100.000 & 100.340 & 0.340 & 2.499 & 99.12 & 100.3094 & 1.18944 \\
\hline 10.004 & 66.810 & 67.037 & 0.227 & 10.099 & 75.52 & 76.2752 & 0.7552 \\
\hline
\end{tabular}

Table 2 : Wedge field beam profile $\%$ of deviation of RTPS data vs measured data

\begin{tabular}{|l|l|l|l|l|l|l|l|l|l|}
\hline \multicolumn{1}{|c|}{ Scanning distance / \%of dose } \\
\hline-68 & -66 & -61 & -56 & -51 & -49 & -46 & -43 & -40 & -37 \\
\hline-0.29 & -0.05 & 0.08 & -0.46 & 0.38 & 0.28 & -0.39 & -0.96 & 0.29 & -0.15 \\
\hline
\end{tabular}

\begin{tabular}{|l|l|l|l|l|l|l|l|l|l|}
\hline \multicolumn{10}{|c|}{ Scanning distance / \%of dose } \\
\hline 37 & 40 & 43 & 46 & 49 & 51 & 56 & 61 & 66 & 68 \\
\hline-0.38 & -0.52 & -1.11 & -1.14 & -0.88 & -0.72 & -1.03 & -1.75 & -0.67 & -0.75 \\
\hline
\end{tabular}

\begin{tabular}{|l|l|l|l|l|l|l|l|l|}
\hline \multicolumn{1}{|c|}{ Scanning distance / \%of dose } \\
\hline-34 & -30 & -20 & -10 & 0 & 10 & 20 & 30 & 34 \\
\hline 0.01 & -0.04 & -0.35 & -0.08 & -0.47 & -0.33 & -0.71 & -0.95 & -0.47 \\
\hline
\end{tabular}

As mentioned above, the overall inaccuracy in dose delivery should not exceed 5\%. Before we started to use TMS in routine work, this point was checked and the error in the output factor or dose calculation was estimated to be less than that level. In the first test we checked the reproducibility of both patient and machine data and we got a near 100\% accuracy result. This test is quite essential because any error in the patient or equipment data may lead to a mistake in the treatment setup. In tests 2 and 3, we found that the calculated output factors were in excellent agreement with measurements made in most cases where the error was always less than $1 \%$. The maximum error $(1 \%)$ occurred in the $60^{\circ}$ wedged fields with maximum field size $\left(20 \times 20 \mathrm{~cm}^{2}\right)$ in $10 \mathrm{MeV}$ beam. This field has a rare clinical application. Tests 4 and 6 showed that at depths larger than the depth of maximum dose, there was good agreement between calculated and measured depth doses and dose profiles[2]. On the other hand, the difference between calculated and measured doses above the depth of maximum dose was higher. For field sizes $10 \times 10 \mathrm{~cm}^{2}$, the error was in the acceptable range while it was not acceptable for smaller fields. There are two reasons for that high error, one of them is due to inaccuracy in dose measurements in the build up region because of the ionization chamber size $(0.1 \mathrm{cc})$. Further measurements in that region with a smaller chamber may give more accurate results. The other reason of the large error is the difficulties in the modeling of the electron contamination[5]. It is known that error due to modeling of electron contamination is higher for 
larger fields and higher energies. In our work, it was found that the error in the build up region is acceptable $(2-3 \%)$ in large fields $\left(>10 \times 10 \mathrm{~cm}^{2}\right)$. Nevertheless, for smaller fields, the error was sometimes not acceptable (3-9\%). This means that for these fields, high error is mainly due to dosimetry inaccuracy. Further dosimetry for fields smaller that $10 \times 10 \mathrm{~cm}^{2}$ was done at depths above depth of maximum dose. We found that the difference between measured and calculated dose was higher than $5 \%$ in only fields smaller than $7 \times 7 \mathrm{~cm}^{2}$. In clinical application, doses in the build up region for fields $\leq 7 \times$ $7 \mathrm{~cm}^{2}$ should not be evaluated from dose calculation by the TMS unit[3]. In test 5 , the calculated and measured star profiles of wedged fields were compared for all energies with the maximum field size. They were in excellent agreement $(<1 \%)$ because of the modification of the calculating model in that version. Previous versions showed less agreement especially for the $60^{\circ}$ wedge.

\section{CONCLUSION}

Dose calculation using TPS based on AAA beam model is generally in excellent agreement with measurements. The only deviation which was not within the limits that have been set up for dose planning systems is the dose calculation above the depth of maximum dose in fields smaller than $5 \times 5 \mathrm{~cm}^{2}$. The reason of that deviation is the uncertainty in dose measurement in the build-up region using $0.1 \mathrm{cc}$ ionization chamber. Additional dosimetry might be done in the region above the depth of maximum dose. It is concluded that implementation of an AAA beam based TPS is suitable for accurate radiation therapy treatment planning and its practical use will decrease the uncertainty in radiotherapy and improve the quality of the feature of radiotherapy planning for the treatment cancer patient.

\section{REFERENCES}

1. Technical reports series no. 398, absorbed dose determination In external beam radiotherapy:an international code of practice for dosimetry Based on standards of absorbed dose to water international atomic energy agency Vienna, 2000.

2. ICRU: Determination of absorbed dose in a patient irradiate by beams of $\mathrm{X}$ or gamma rays in radiotherapy procedures. ICRU Report, 24, 1976.

3. Technical reports series no. 430: International atomic energy agency Vienna, Commissioning and quality assurance of computerized planning systems for radiation treatment of cancer, 2004.

4. American Association of Physicists in Medicine (AAPM) Radiation Therapy Committee Task Group 53: Quality assurance for clinical radiotherapy treatment planning, Received 15 December 1997; accepted for publication 4 August 1998.

5. AAPM's TG-51 protocol for clinical reference dosimetry of high-energy photon and electron beams: Received 17 September 1998; accepted for publication 4 June 1999.

6. Brahme A.: Dosimetric precision requirements in radiation therapy. Acta Radiol. Oncol., 23: 379- 91, 1984.

7. ICRU: Use of computers in external beam radiotherapy procedures with high energy photons and electrons. ICRU Report, 42, 1987. 